\title{
LES RELATIONS CULTURELLES DE LA TURQUIE AVEC LES PAYS DE LA COMMUNAUTE EUROPEENNE*
}

— La vision culturelle européenne de la Turquie -

Dr. Ali Engin OBA

Le 14 avril 1987, le gouvernement turc a officiellement déposé la demande d'adhesion de la Turquie à la Communauté européenne.

Le Conseil, le 27 avril 1987, en prenant acte de cette demande, a prié la Commission de rendre l'avis requis par les textes en vigueur. Cet avis fut divilgué le 14 décembre 1989.

La Grande partie de ce document s'arrêtait sur les problèmes économiques et pólitiques de la Turquie et l'avis précisait qu'à ce stade, la communauté étant en pleine evolution dans une Europe en mutation, il était inopportun de procéder à de nouvelles négociations d'adhésion; en outre, le contexte économique et politique de la Turquie a conduit la Commission a estimer qu'il ne serait pas utile d'entamer dès maintenant l'ouverture de négociations d'adhésion avec le pays en question. D'après ce document, la Commission estimait cependant que la Communauté devait poursuivre sa coopération avec la Turquie, étant donné l'orientation générale de ce pays vers l'Europe (1).

Le dernier paragraphe de l'avis était consacré a la culture et formation: "Il serait également opportun d'approfondir les liens en matière de formation et de culture entre la Communauté et la Turquie en vue de contribuer à une meilleure compréhension réciproque; à cet effet, la Turquie pourrait utilement étre associee à certains programmes communautaires".

L'avis de la Commission était donc basé plutôt sur les questions économiques et politiques de la Turquie. Un seul petit paragraphe consacré à la

\footnotetext{
* Cet article engage la seule responsabilité de l'suteur et nullement celle du ministère turc des Affaires Btrangères.
} 
culture et a la formation ne montrait -il pas que la dimension culturelle de la demande d'adhésion de la Turquie à la Communauté ne constituait pas un problème.

Bien qu'elle soit difficile a définir, on peut dire que la culture est l'ensemble des oeuvres, des réalisations, des institutions qui déterminent l'originalité et l'authenticité de la vie d'un groupe humain (2). Plus simplement, on peut dire que la culture est l'ensemble des traits distinctifs qui caractérisent une société ou un groupe social (3).

D'après Edgar Morin, la culture européenne est judéo-christiano-grécolatine: les sources juives, chrétiennes, grecques, latines ont formé une synthése qui a constitué le dénominateur culturel commun sur lequel l'Europe a produit une civilisation originale marquée par l'humanisme, la spiritualite, la rationalisation et la démocratie (4).

Selon Hans Sand, qui a préparée le document de séance de la 6ème conférence des Ministres européens responsables des Affaires culturelles, il serait impossible de définir la culture européenne; certes, on peut parler de culture, d'une base culturelle européenne creant ainsi l'aspect pluraliste du patrimoine culturel européen (5), Il conclut après cette analyse que la culture européenne est plus une mosaïque de cultures qu'une culture unique (6).

Carlo De Benedetti, Président de Olivetti, dans son discours prononcé au Collège d'Europe, après avoir expliqué l'importance de l'épanouissement culturel pour la société européenne du XXIème siécle, a préconisé la diversité des cultures: "La diversité des cultures represente pour l'Europe non pas une faiblesse, mais un atout extraordinaire qui doit étre protége et grâce auquel pourront étre construites des complémentarités positives et une dialectique fondée sur la créativité et linnovation continue" (7). Il estime que la dimension culturelle de l'Europe "doit devenir un point de référence constant dans la construction de la nouvelle Europe" (8). Il pense que l'Europe est a méme de produire une culture pour le XXIème siécle, "une culture qui se fonde sur la liberté, sur le pluralisme, sur la force de la diversite, mais aussi sur l'utilisation complète et intelligente des technologies de l'information" (9).

Sand décrit la principale caractéristique de la culture européenne contemporaine comme une attitude commune à l'egard de valeurs telles que ouverture d'esprit, la tolérance, les droits et les libertés civiles, le respect de la vie et la croyance dans le progrés et l'utilité des réformes (10).

La Charte de Paris pour une Europe nouvelle, adoptee a l'issue de la 
conférence sur la sécurité et la coopération en Europe, souligne également la diversité de la culture europeenne (11).

Donc, on peut déduire de ce qui précède qu'il est difficile de définir les éléments qui forment l'identité culturelle européenne et c'est la raison pour laquelle la société européenne est concue comme une société multiculturelle (12).

D'ailleurs, la déclaration sur la "société multiculturelle et l'ldentité culturelle européenne", adoptée à l'issue de la 6ème conférence des Ministres européens responsables des Affaires culturelles, affirmait que la richesse de la culture européenne dérivait de la diversité et de la vitalité de ses cultures nationales, régionales et locales ainsi que de son ouverture aux courants spirituels, intellectuels et artistiques venant des autres parties du monde (13). Un autre article de la même déclaration prévoyait que les Européens se doivent a la fois de préserver et de promouvoir ce qui les différencie les uns des autres, compte tenu de la richesse que représente le pluralisme des expressions culturelles (14).

Sand, dans son document de séance, ne manquait pas de préciser que la base culturelle européenne avait été fortement influencée par des cultures non européennes. Il ajoutait que c'était vrai pour l'héritage religieux(15). Il soulignait également la contribution du proche et du moyen Orient ainsi que l'Afrique du Bord sur la culture européenne qui a été marquée par leurs influence (16).

Thierry Hentsch écrivait sur le rôle des intellectuels et de la culture dans la construction politique de l'Europe: "Dégagée de sa position hégémonique, l'Europe actuellement profite de sa faiblesse relative pour penser son désarroi à travers les désarrois plus vifs, mais peut-être plus créateurs d'autres cultures, en particulier de cette Méditerranée musulmane qu'elle s'est trop longtemps contentée de rejeter" (17).

Le Professeur Jacques Berque, rapporteur général de la conférence pluridisciplinaire sur les aspects éducatifs et culturels des relations intercommunautaires, tenue les 5-7 décembre 1989 a Strasbourg, commençait son rapport en indiquant que l'Europe de la prochaine décennie débattra sans doute de problémes culturels aujourd'hui partiellèment occultés par les problèmes économiques et sociaux (18). Il précise son point de vue par la phrase suivante: "Certains problèmes surgiront d'une concurrence entre les cultures dominantes; d'autres de la contestation de celles-ci par des entités régionales, minoritaires ou refoulées; d'ailleurs avec les immigrés. Ces cultures dites ha- 
bituellement d'origine, je propose de les appeler cultures d'apport. Leur contribution pourrait étre en effet positif pour les uns et les autres, à condition d'aménager entre les parties concernées des relations fondées sur le réalisme et les droits de l'homme" (19).

Eu égard à l'aspect multiculturel de l'Europe, la Turquie moderne, läque, ayant une base culturelle islamique, restee partiellement en dehors de la génèse culturelle européenne, a-t-elle une place dans cette société multiculturelle qu'est l'Europe?

\section{La vision culturelle européene de la Turquie}

Edward Mortimer, dans la Communication qu'il avait faite au cours du symposium International sur l'identité culturelle européenne qui avait lieu à Paris le 13-14 janvier 1988, après avoir précisé que l'appartenance de la Turquie à l'Europe était déjà admise, n'hésitait pas pourtant à souligner la base de l'identité européenne provenant de l'héritage chrétien et de l'Antiquité gréco-romaine; et il posait la question capitale: "... comment un pays dont l'herritage est pour l'essentiel tslamique v-t-il réellement s'y associer et comment les citoyens des autres pays européens vont-ils pouvoir sidentifier à une communauté dans laquelle la Turquie ne serait-ce que par son poids démographique, serait appelée à étre l'un des membres dominants?" (20)

M. Murat Dikmen, l'unique personnalité turque présente à cette réunion, éprouvait la nécessité de répondre à l'intervention de M.Mortimer: "Il est bien vrai qu'il y a d'autres componsantes (et plusieurs l) de la culture. Mais si l'on se bome en particulier à l'une d'elles, par exemple à la composante religieuse vers la fin du XXème siècle, tant d'années après ce qui fut connu comme le siècle des Lumière, on ne peut s'empècher de se demander s'il s'agit là toujours d'un critère ou plutôt d'un souci de refuge. Permettez-moi de ne pas conclure." (21)

Dr. Udo Steinbach, dans sa communication sur la dimension culturelle des relations de la Turquie avec la Communauté, adoptait une attitude mitigée à l'égard de la culture turque (22). Il estimait que le développement culturel de la Turquie était dirigé davantage vers une synthèse (23). Il attirait l'attention sur la laiicité et lançait un appel aux élites turques: Les membres de l'élite turque qui désirent avoir une place dans l'Europe doivent définir sans hésitation où ils se trouvent culturellement. (24)

Sir Bernard Burrows reflète le même sentiment en indiquant qu'il serait 
difficile de faire accepter facilement a l'opinion publique des pays européens que la Turquie partage avec eux la méme base culturelle (25).

Ce stéréotype persiste en dépit de l'article 15 de la Declaration européenne sur les objectifs culturels, adoptée par la 4éme Conférence des ministres européens responsables des Affaires culturelles (Berlin 1984) qui stipule la création des conditions favorables à une meilleure compréhension entre populations, d'âges et de cultures, de religions et de traditions différents (26).

Même la parution d'un livre impartial, basé sur des documents d'archive, sur l'Empire Ottoman, suscitait la réaction de certains milieux désirant voir toujours la validité de ce stéréotype (27).

Pourtant, l'historien Braudel, avant même la publication de ce livre, avait accepté les richissimes archives turques révèlant la reelle grandeur turque : "Peu à peu, cette réelle grandeur turque, déniée après coup, émerge grâce aux recherches des historiens: les richissimes archives turques, enfin classées, commencent à s'ouvrir aux chercheurs et révélent, un à un, les rouages d'une bureaucratie multiple, précise, progressiste, autoritaire, capable de deresser des recensements détaillés, de concevoir une politique intérieure cohérente, d'amasser d'énormes réserves d'or et d'argent, de coloniser systématiquement (en y établissant des nomades) les Balkans, bouclier de l'Empire, face à l'Europe. Un systéme de travail forcé, une armée étonnante avec de durs apprentissages... En vérité, d'étranges modernités" (28).

Jean d'Ormesson, après un bref séjour en Turquie, écrivait ce qui suit: "Les civilisations, en Turquie, se bousculent les unes les autres, s'exterminent et coexistent. On dirait les cartes d'un jeu cosmique battues par un géant. Le voyageur qui, comme moi, passe quelques jours en Anatole voit défiler devant ses yeux les millénaires et les cultures. A la façon d'un plongeur qui passerait a toute allure d'une profondeur à une autre, il est trimbalé en un clin d'oeil de l'art grec le plus classique aux splendeurs ottomanes ou seldjoukides, des pères de l'Eglise aux mystiques musulmans, de l'aube de la culture urbaine aux raffinements d'une civilisation nourrie à toutes les sources de l'Orient et de l'Occident" (29). Donc, d'après d'Ormesson, la culture turque présente le caractère d'une synthèse provenant des sources orientales et occidentales.

Et si on prend en considération l'Anatolie, berceau de plusieurs civilisations possédant un patrimoine d'une grande richesse, peut-on penser a la culture europénne sans Anatolie? Comme c'est très bien décrit dans l'ouvrage 
de M. Turgut Ozal, "La Turquie en Europe", l'Anatolie est une partie intégrante de la culture occidentale (30). Sans la Turquie, la culture européenne ne sera pa sreprésentée intégralement.

Et les Balkans? L'Empire Ottoman était present en Europe depuis le XIVème siécle, quand la Turquie s'est établie sur la rive occidentale de la mer de Marmara, en vue de s'étendre ensuite sur les Balkans. La présence européenne de la Turquie a duré un peu plus de six cents ans, rendant ainsi possible une longue cohabitation avec les peuples de l'Europe du sud-Est. Les valeurs communes dont se réclament les pays de cette demière région étaient le produit d'un patrimoine culturel, diversifié aux époques de forte influence méditerranéenne, gréco-latine, byzantine et ottomane (31). Et, d'après Dimitri Kitsikis, l'Europe culturelle n'a jamais été une. Il prétend qu'il y a toujours eu deux Europes politiques, "celle centrée sur la mer Egée et la ville d'Istanbul et celle centrée sur l'axe RomeVienne" (32). Suivant la ligne de ce partage, il pense qu'on a pu distinguer "deux entités culturelles européennes, une Europe germano-latine et catholico-protestante à l'Ouest, une Europe slavo-gréco-turque et chrétienne-orthodoxe-musulmane à l'Est" (33). Il va encore loin lorsqu'il précise ce qui suit: "Si la Grèce et la Russie sont des pays européens, alors la Turquie l'est également ou alors, aucun des trois pays ne l'est" (34). Il estime que l'idée d'un lien nécessaire entre christianisme et Europe est un mythe (35). Il met en avant l'idée suivante: "Au cours des siècles, il s'est avéré qu'il y avait une grande différence entre orthodoxie et catholicisme, plus qu'entre orthodoxie et islam (les Grecs chretiens orthodoxes se sont toujours mieux entendus, sur le plan religieux, avec les Turcs musulmans qu'avec les Latins catholiques). Ensuite, parce que la deuxième Europe, celle de l'Est, a depuis des siécles été habitée par d'importantes populations musulmanes que même les échanges de population du XXème siècle n'ont pas réussi à éliminer: pensons a l'actuelle Albanie, à la Yougoslavie, a la Bulgarie ou à la Russie" (36). Et il lance l'appel suivant à la Communauté: "Si l'ambition de la Communauté Economique Européenne est de répéter l'exploit de l'Empire d'Auguste du temps de Jésus, à savoir d'unir deux Europes qui, jusqusau XXème siecle du moins, étaient si dissemblables et puisque cette Communauté a déjà accepte la Gréce orthodoxe en son sein, par quelles logiques pourrait-elle refuser l'alter ego de l'orhodoxie grecque, qui est l'Tslam turc" (37)?

Il résulte de ce qui précède que l'ignorance et les préjugés sur la culture turque dans le monde europén sont les éléments principaux falsifiant l'image turque à travers l'Occident. Cette image est une invention de l'Eu- 
rope. On peut dire avec Edward Saìd qu'elle est la representation que l'Europe se fait de l'Orient. Mais, "l'Orient est partie intégrante de la civilisation et de la culture matérielles de l'Europe" (39). Cette image est l'oeuvre de la vision politique occidentale de l'Est méditerranéen (40). Cet Orient imaginaire qui constitue l'antithèse de la modemité, est la source des idées, des clichés défavorables qui influent sur la vision culturelle europé Turquie. $\mathrm{La}$ responsabilité de cette situation incombe partiellement aux autorités turque à l'étranger sur la Turquie en général et sur sa culture en particulier, mais en méme temps "à un travail de désinformation méthodique, parallèle aux enjeux politiques et économiques menés sur l'échiquier international" (41). En outre, il ne faut pas oublier la littérature européenne disséminant également l'image négative d'une Turquie qui serait l'ennemi de la chrétienté (42). Ces préjugés continuent à exister aujourd'hui en depit de la parution du livre de M. Mantran sur l'empire ottoman cité ci-dessus. En effet. M. Gérard Slama, dans son article publié dans la revue "Le Point" n'hésitait pas à accuser le livre de M. Mantran de manque d'objectivité (43). La réponse de M. Mantran a cette critique constituait sans doute la réfutation exemplaire de ces préjugéssur la Turquie et les turcs existant en Europe: "Si I'histoire de l'Empire ottoman, qui se veut une mise au point sincere et sans parti pris, peut apparaitre comme favorable aux ottomans et aux Turcs, c'est que depuis cent cinquante ans, notre éducation historique a été marquée par la méconnaissance de cette histoire vue au travers des images simplistes et parfois fallacieuses, marquée aussi par le triomphe d'une Europe occidentale seule capable d'apporter les lumières de la civilisation" (44).

\section{La Turquie et la Culture Européenne}

Les relations culturelles de la Turquie avec les pays de la Communauté sont très anciennes. Un bref regard jeté sur l'historique dé ces relations prouvent un intense dialogue culturel entre les differents pays européens et la Turquie provenant de divers contacts datant du XVIème siècle, époque où l'Empire ottoman était une superpuissance. Les guerres, le commerce et l'orientalisme du a l'interet des pays europeens pour tout ce qui venait de l'orient et surtout de la Turquie constituait sans doute les vraies sources d'un vif intérêt intellectuel en ce qui concerne l'ant et la culture turco-ottomane (45).

L'Empire ottoman, de son côté depuis la promulgation le 3 novembre 1839 du l'rescrit impérial de Gülhane (Tanzimat), est entré dans une ére nouvelle dans laquelle un vaste programme de réformes visant à moderniser et à 
occidentaiiser la sociéte et l'administration ottomane a vu le jour (46). L'évolution économique et sociale a transformé l'appareil d'Etat. Le changement a touché également la société toute entière et en a modifié les fondements culturels.

La culture turco-ottomane était originale et riche, provenant d'une vaste synthèse basée sur la culture des Turcs et des populations soumises aux Ottomans coexistant dans ce vaste empire (47). Louis Bazin estimait que les influences de l'Europe chrétienne étaient minimes et que le sentiment de solidarité avec les Arabes musulmans n'a pas frayé la voie à l'arabisation de la culture turque (48).

La période des Tanzimat (1839-1878) a joué un rôle important dans la pénétration de la culture européenne dans l'Empire. L'apparition du concept de modernité parmi les dirigeants de l'Etat, les réformes entreprises par le Sultan Mahmut II et son fils le Sultan Abdul-Medjid (1839-1861) étaient sans doute le prélude d'un vaste changement vers la modernisation et l'occidentalisation de l'Empire. La langue française était devenue la seconde langue officielle de l'Empire véhiculant ainsi la pénétration de la culture, des idces et des modes françaises dans les classes dominantes et chez les intellectuels (49). La diffusion de la culture occidentale n'a pas cessé de répandre dans l'Empire, les efforts de modernisation dans tous les domaines de la vie sociale et intellectuelle et ont continué sans cesse par l'apparition de la presse et la création de l'enseignement moderne calqué sur le système français. L'impact de la littérature française sur la littérature turque, la création du roman et du theatre moderne turc, l'occidentalisation des arts musicaux avec la venue d'orchestres européens, la maitrise d'instruments et surtout du piano, l'introduction de la peinture a l'huile, l'occidentalisation des arts décoratifs et architecturaux ont constitué les autres éléments de l'influence culturelle européenne sur la Turquie (50).

Cette culture occidentale a coexisté avec la culture traditionnelle sans la faire disparaitre. Cette situation dualiste a dure jusqu'a la proclamation de la république. Les réformes entamées par Atatürk ont visé à donner à la Turquie tous les eléments d'un pays modeme, occidental en mettant fin a la situation dualiste. Les reformes en question avaient comme but l'adoption de la culture contemporaine occidentale (51). L'adoption de l'alphabet latin et du laicisme ainsi que de toutes autres institutions europeennes en matière d'organisation d'Etat-nation turc, de la démocratie pluraliste, constituaient d'occidentalisation complete de la Turquie et lui octroyaient une place parmi les pays européens. Le laicisme avait une importance particulière car il marquait la rup- 
ture avec le passé ottoman et le caractère islamique du régime de l'Empire (52).

La création du conservatoire d'Etat à Ankara, l'arrivée des pédagogues, des artistes étrangers comme Joseph Marx, Paul Hindemith, Bela Bartok, Eduard Zuckmayer en Turquie en 1932, l'envoi des jeunes étudiants turcs en Europe en vue d'étudiler ou bien de perfectionner leurs connaissances dans le domaine de l'art, notamment de la musique, de la peinture, du theàtre et de l'architecture ont aidé à occidentaliser la riche culture turco ottomane (53). La jeune république kemaliste a pu creer les conditions qui ont permis a la culture contemporaine turque, basée sur la synthèse de son passé, de se répandre d travers les classes sociales.

Aprés la deuxième guerre mondiale, ce courant a encore pris un élan plus vaste avec l'ouverture complete de la Turquie à l'Occident. L'introduction de la démocratie multipartite l'adhésion de la Turquie en tant que membre à part entière de toutes les organisations européennes, telles que l'OTAN, le Conseil de l'Europe et l'OCDE ont accru la conscience européenne des Turcs et leur appartenance à la civilisation contemporaine $o c$ cidentale. Les liens étroits avec l'Europe ont joué un rôle cardinal dans le développement des relations culturelles et d'établissement d'accords culturels. Les programmes basés sur ces accords ont contribué a promouvoir les contacts culturels de la Turquie avec les pays de la Communauté européenne. D'autre part, les Gouvernements turcs ont également pris des mesures nécessaires en vue de la promotion de ces relations. En effet, le programme du gouvernement actuel a prévu le développement des liens culturels avec les pays de la Communauté.

Comme nous l'avons précisé auparavant, les programmes culturels basés sur les accords ont constitue le cadre des activités culturelles entre la Turquie et les pays de la Communauté. En guise d'example, je voudrais résumer le contenu du dernier programme culturel turco-français, élaboré après la réunion de la Commission Mixte de Coopération Culturelle Scientifique et Technique turco-française qui s'est rénie à Ankara du 20 au 22 juin 1988, conformément à l'accord culturel du 17 juin 1952 et à l'accord de coopération scientifique et technique du 29 octobre 1968. Le programme en question, comme tous les autres programmes culturels, a été conclu pour une durée de deux ans.

Conformément aux décisions prises par les autorités responsables turcofrançaises et en vue de développer la coopération dans les domaines de la 
culture et de l'enseignement, les deux pays ont étudié les sujets évoqués cidessous et ont convenus des moyens suivants a mettre en oeuvre (54).

I- Echanges culturels et artistiques
A. Arts plastiques
- Exposition Soliman le Magnifique
- Exposition de lithographies turques dans trois villes de France dont Paris
- Participation de la France au Festival d'Tstanbul en 1989 a la Biennale d'Ankara en 1990
- Organisation d'une exposition de peinture contemporaine turque en France
- Présentation de deux expositions d'art contemporain français en Turquie

\section{B. Arts de la scène}

Les deux parties conviennent de favoriser une participation régulière d'artistes et de groupes français au Festival International d'art et de la culture d'Tstanbul:

- Participation de l'Orchestre National de Lyon au Festival d'Istanbul en 1988

- Participation de 17 musiciens turcs à l'Orchestre des Jeunes de la Méditerranée en 1988

- Projet de mise en scéne d'une pièce française au Théatre National d'Ankara avec Charles Tordjmann, directeur du Théatre Populaire de Lorraine

- Projet de montage de l'Opéra-Ballet Padmavati d'Albert Roussel

- Projet de réalisation pour l'ouverture de la saison d'opéra 1989 90 d'Tstanbul de l'Opéra Faust de Gounod en langue française

- Projet de réalisation d'un concerto de piano de Bülent Tarcan par l'orchestre de Radio-France sous la direction d'Erol Erdinç et participation de Hülya Tarcan comme soliste 
- Reconduction de l'invitation d'un artiste ou d'un metteur en scéne du Theare d'Etat turc au Festival d'Avignon

- Envoi chaque année par la partie française de quelques groupes de musique et de théătre en Turquie

- Offre par la partie française, chaque année, de 26 bourses dans le secteur artistique

\section{Arts et spectacles traditionnels}

Les deux parties ont convenu de favoriser réciproquement des échanges de groupes folkloriques.

D. Cinéma

- Coopération en matière de dessins animès, archives de films et autres domaines

- Participation aux festivals de films organisés dans les pays respectifs, la partie française manifeste son intérêt pour la venue en France d'un metteur en scène turc confirmé.

\section{E. Radio-Télévision}

Les deux parties ont convenu de développer la coopération entre les organismes de télévision des deux pays et l'échange de programmes télévisés.

\section{F. Musée et Archéologie}

Les deux parties ont convenu d'encourager la collaboration dans les domaines de muséologie, protection et restauration des monuments historiques.

G. Documentation, publications et bibliotheque

- Echange de livres, de micro-films et de periodiques entre les Bibliothéques Nationales des deux pays et collaboration dans le domaine de la conservation des manuscrits:

- Participation réciproque aux expositions et foires du livre qui seront organisées dans les deux pays. 


\section{H. Commémorations}

Les deux parties ont convenu d'organiser et de faciliter les activités de commémoration qui seront entreprises a l'occasion du 50ème anniversaire de la mort d'Ataturk, du 200eme anniversaire de la Révolution Française, du 100גme anniversaire de la naissance de de Gaulle.

II- Activités dans le domaine de la Jeunesse, contacts, sports et échanges

III- Coopératión dans le domaine de l'Eduction

1. Problèmes d'enseignement des enfants de travailleurs turcs en France.

3. Enseignement de la langue turque dans les institutions d'enseignement et de recherche en France.

4. Enseignement du Français dans les établissements scolaires en Turquie: Lycée de Galatasaray, Lycée Anatolien d'Ankara; formation de professeurs turcs de Français en France; départements de Lettres et de Langues dans les universités; étude de la methodologie du Français dans l'enseignement secondaire.

5. Allocation de bourses

La partie turque bénéficiera d'un nombre important de bourses linguistiques et de recherches, d'études de formation universitaire de 3ème cycle.

6. Coopération interuniversitaire

Les deux parties ont convenu da faire le nécessaire pour améliorer la cooperation interuniversitaire entre les deux pays.

IV- Moyens de développer une atmosphère de compréhension et d'estime mutuelle entre les deux peuples.

Les deux parties proposent qu'une attention soit portée a l'enseignement de l'histoire et de la géographie dans les manuels scolaires et les Atlas.

V-Coopération scientifique et technique

1. Création de facultés et départements francophones dans les universités turques: 
- Création d'un département francophone de Sciences politiques et administratives à l'Université de Marmara

- Création d'un programme d'enseignement universitaire du 3ème cycle en Français au departement de Gestion de la faculté de Sciences Economiques et Administratives de l'Université de Hacettepe

2. Projets d'intérét commun dans diverses universités portant sur l'informatique, la biologie, l'hydrobiologie, l'aéronautique, l'agronomie et industrie agricole, et la sante.

3. Recherche scientifique: coopération C.N.R.S.-TÜBITAK (55).

Les deux parties apprécient la reprise des relations qui s'amorce entre TÜBITAK et le C.N.R.S.

\section{CONCLUSION}

Nous avons tâché d'exposer comment la Turquie et la culture turque ont été perçues par les Européens en vue de répondre à la question de savoir si la Turquie moderne laịque ayant une base culturelle islamique, restée partiellement en dehors de la génèse culturelle européenne, mais ayant opté définitivement pour cette dernière il y a presque 70 ans, a une place dans cette Europe des Douze qui est une société multiculturelle.

Il y a plus de 150 ans, la Turquie ottomane et puis moderne s'est orientée vers l'occident en acquérant les valeurs européennes. La dernère partie de cette période, c'est-à-dire les 70 dernières annèes a constitué une ère où la culture européenne s'est implantée dans la vie quotidienne du pays.

Appartenir a la culture contemporaine est devenu le but ultime des réformes d'Atatürk et cet héritage est passé de génération en génération avec une identité culturelle bien établie se nourissant d'une civilisation ancienne et en lui donnant une accumulation culturelle multidimensionnele (56). Il faut ajouter à cette dernière l'histoire commune de plusieurs siècles provenant de la cohabitation puis de relations étroites avec les pays occidentaux: tout ceci ajoute fait de la culture turque une synthèse originale. Cette synthèse qui est le compromis entre la civilisation orientale et occidentale reflete lidentite culturelle turque (57). Cette identité n'est pas une imitation de l'Occident ou une adoration de valeurs anciennes qui seraient dépassées. C'est une culture riche, englobant la synthèse des cultures judéo-chrétienne-islamique et 
gréco-latine-ottomane, qui constitue sans doute une partie de la culture européennes. Prétendre le contraire, c'est ne pas prendre en considération l'Europe du Sud-Est et la méditerranée comme partie integrante de la civilisation et de la culture occidentale, Cela résulte d'une mauvaise connaissance de la Turquie ou de prejugés historiques qui consistent à voir ce pays sous un angle plutôt religieux. En outre, l'Occident, comme c'est bien exprimé par le professeur Mantran, a toujours eu de la Turquie une perception simpliste et parfois fallacieuse marquée par le triomphe d'une Europe qui se croyait seule capable d'apporter les lumières de la civilisation. L'ignorance de l'Occident à l'épard de la culture turque dérive du manque d'information adéquate à e'étranger (58).

La principale caractéristique de la culture européenne contemporain peut être exprimée une même attitude à l'égard de valeurs telles que l'ouverture d'esprit, la tolérance, les droits et les libertés civiles, le respect de la vie et la croyance dans le progrès at l'utilité des réformes. La Turquie respecte ces valuers, les partage et tâche de les promouvoir; sa constitution les prend en compte... Adoptant ces valeurs la Turquie devient partie intégrante de l'Europe.D'ailleurs, son passé, "pax ottomana", est témoin de la tolérance et du respect de la vie et de la foi. Les juifs opprimés et exécutés en Espagne ont trouvé refuge en Turquie. Les Suédois, les Hongrois, les Polonais et les autres n'ont eu aucune difficulté en demandant l'asile politique aux autorites turques. Le Turquie moderne poursuit le même chemin et tâche d'harmoniser son système judiciaire à celui de la Communauté. En outre, avec son développement économique, elle est en train de combler son retard par rapport aux pays industrialisés. Le progrès économique lui apportera les bases socio-culturelles facilitant son adhésion à la Communauté. La richesse de sa culture constituera sans doute un atout important pour l'accomplissement de ce processus.

Bien que la Turquie ait une population en majorité musulmane, elle est un état laïque. L'Europe larique ne doit pas creer de problème en vue de l'adhésion de ce pays en son sein en raison de différences de religion. L'adoption d'une telle attitude serait une décision malheureuse qui traduirait une position partiale et non fondée de l'Europe face a la Turquie. Il ne faut pas non plus perdre de vue les caractéristiques communes de deux religions. L'Tslam en tant que dernière religion monothéiste vénère le Christ comme un prophète. Il est fortement impressionné par la civilisation gercque antique et a constitué un pont entre la civilisation européenne. La Turquie participe activement au dialogue de deux religions dans les forums internationaux en vue 
de créer une base facilitant la compréhension mutuelle.

En somme, la Turquie est en train d'entreprendre tout ce qui est en son pouvoir afin de créer une conscience culturelle européenne parmi ses citoyens et de promouvoir à l'étranger l'image d'un pays partageant les valeurs culturelles et sociales de l'Occident. Ces actions aplaniront tous les obstacles qui s'opposent à son adhésion à la Communauté.

\section{NOTES}

(1) Avis de la Commission sur lademande d'adhésion de la Turquie a la Communauté; Bruxelles, le 4 décembre 1989, P. 8.

(2) Victor Hell, L'idée de Culture, Paris, coll. "Que sais-je" 1981, p. 51.

(3) Guide Pratique de la Décennie Mondiale du Développement Culturel, Paris, Unesco, 1987, p. 5.

Pour une analyse sociologique de la culture, voir: Raymond Williams, Culture, Fontana Paperbacks, 1981, p. 9-32.

(4) Edgar Morin, Penser l'Europe, Paris, Gallimard 1987/71

(5) Hans Sand, Document de Séance de la 6ème Conférence des Ministres Européens responsables des Affaires Culturelles Palerme, Italıe 25-26 Avril 1990, p.1.

(6) Ibid.

(7) M. Carlo de Benedetti, Président de Olivetti, Le Management Europén face au XXIème siècle, Discours au collège d'Europe, Bruges, le 24 novembre 1989, texte du discours p. 11.

(8) Ibid., p. 17.

(9) Ibid.

(10) Sand op. cit., p. 2.

(11) Charter of Paris for a New Europe, November 1990, p. 14.

(12) Le sujet de la 6 ème conférence des Ministres européens était consacré a la société multiculturelle.

(13) Déclaration, société multiculturelle et identité culturelle européenne, 
article 3.

(14) Ibid., article 7.

(15) Sand, op. cit. p. 1

(16) Ibid.

(17) Thierry Hentsch, "L'usage de l'autre dans la construction d'un mythe de l'Europe (a propos du rôle des'intellectuels et de la culture dans la construction politique de l'Europe)", Du Personnalisme au Fé-

déralisme Européen, en hommage à Denis de Rougemont, Edition centre Européen de la Culture, 1989, p. 239.

(18) Jacques Blerque, Synthèse des travaux "Comment permettre à des citoyens d'origine diverse de participer à une société démocratique et plurielle", p. 1.

(19) Ibid.

(20) Edward Mortimer, "Sur quels critères peut-on décider qu'un pays est européen ?", Europe Sans Rivage De l'Tdentité culturelle Européenne, Paris, Albin Michel, 1988, p. 250-251.

(21) Murat Dikmen, Ibid, p. 293.

(22) Dr. Udo Steinbach, "Turkey-EEC Relations, Cultural Dimension, International Gime Conferences, Turkey's places in Europe, economic, political and cultural dimensions, Edited Erol Manisalı, Istanbul, 1988, p. 13-16.

(23) Ibid., p. 22

(24) Ibid., p. 23

(25) Sir Bernard Burrous: "Turkey and Europe : The Cultural Background Turkey and Europe in a Cultural Context, Centre of Middle Eastern Studies, University of Cambridge, June 1988, p. 1.

(26) Déclaration européenne sur les objectifs culturels, Strasbourg, 1984, p.4

(27) Robert Mantran (Editeur), De l'Empire Ottoman, Fayard, Paris, 1989.

(28) Fernand Braudel, Grammaire des Civilisations, Paris, Arthaud, Flammarion, 1987, p. 123. 
(29) Jean d'Ormesson, La Chronique du temps qui passe, un berceau et un creuset, Figaro-Magazine, 21 Octobre 1989, p. 9.

(30) Turgut Ozal, La Turquie en Europe, Paris, Plon, 1988, p. 21-90.

(31) Virgil Candea, "Valeurs communes a travers les diversités socioculturelles: le cas de l'Europe du Sud-Est", cadmos, automne/hiver 1989, p. 74.

(32) Dimitri Kitsikis, "Les Turcs et la Mer Egée: Essai de géohistoire", Turquie, Moyen-Orient, Communauté Européenne, l'Harmattoun, Paris, 1989, p. 280.

(33) Ibid.

(34) Ibid.

(35) Ibid.

(36) Ibid.

(37) Ibid., p. 281

(38) Edward Saìd, l'Orientalisme, l'Orient cree par l'Occident, Paris, Svoul, 1978, p. 13.

(39) Ibid., p. 14.

(40) Thierry HENTSCH, L'Orient imaginaire, la vision politique $\alpha c-$ cidentale de l'Est méditerranéen, Paris, Les Editions de Minuit, 1988, p. 9-16.

(41) Pulat Tacar, "Demandes d'adhesion de la Turquie aux Communautés européennes: Motifs politiques turcs et réactions communautaires', Turquie, Moyen-Orient, Communauté européenne op. cit., p. 346.

(42) Hasan Anamur, "Les Réformes d'Atatürk à travers les témoignages des écrivains français contemporains", De la Révolution française à la Turquie d'Atatürk, Istanbul, Isis, 1990, p. 217-218.

(43) Alain Gêrard Slama, Le Point n 908.

(44) R. Mantran, Le Point no 911 courrier.

(45) Cet intérêt a pu même crêer un impact sur les éléves ottomans étudiant en France qui, a leur retour a Istanbul, sont devenus des nationalistes 
ardents et ont joué un rôle assez important dans la génèse du nationalisme turc dans l'Empire ottoman.

(46) Pour Tanzimat: Paul Dumont, La période des Tanzimat (1839-1878), Histoire de l'Empire ottoman, op. cit, p. 459-522).

(47) Louis Bazin, "La vie intellectuelle et culturelle dans l'Empire ottoman", Histoire de l'Empire ottoman, op. cit., p. 695.

(48) Ibid., p. 703.

(49) Ibid., p. 717.

Pour une évaluation de l'orientation vers la culture occidentale, voir: Şerafettin Turan, Türk Kültür Tarihi, Türk Külttüründen Türkiye Kültürüne ve Evrensellige, Ankara Bilgi Yayinevi, 1990, p. 251-306.

(50) Bazin op. cit., p. 724.

(51) Lewis Bernard The Emergence of Modern Turkey . London 1961 p. 273, Özal op. cit., 208-209.

(52) Özal, op. cit., 211.

(53) Metin And, Culture, Performance and Communication in Turkey, Institute for the Study of Languages and Cultures of Asia and Africa, Tokyo, 1987, p. 59-69.

(54) Procés-verbal de la Commission mixte de Coopèration Culturelle Scientifique et Technique Turco-Frençaise, réunie à Ankara les 20-2122 juin 1988, p. 1-2-5.

(55) L'abbréviation de l'organisation turque de recherche scientifique et technique.

(56) Ismet Birsel, Dış Politika öncelikleri ve Kültürel Tanıtım, p. 3, communication présenté à la Deuxième Convention nationale de la Culture, décembre 1989, Ankara.

(57) Ibid.

(58) Pour la promotion de la culture turque à l'étranger, voir: Pulat Tacar, "Türkiye'nin Kültürel Tanıtumı; için oneriler", communication présentée à la Deuxième Convention nationale de la Culture, décembre 1989, Ankara. 